\title{
THIN-PLATE SPLINE ANALYSIS OF SHAPE DIFFERENCES BETWEEN A PRIMITIVE AND MODERN RHINOCEROS.
}

BALES, Gerald S., Department of Anatomy \& Cell Biology, University of Southern California, 1333 San Pablo St., Los Angeles, CA., 90033 U.S.A.

The thin-plate spline (TPS), a new technique for comparing shapes using landmark data, has been developed by Fred Bookstein and programmed for microcomputers by F. Rohlf. TPS generates a mapping function describing the deformation necessary to superimpose one set of landmarks exactly onto a second set of homologous landmarks, after first adusting for orientation and overall size. The mapping function is then used as an interpolant to plot a grid which geometrically represents the deformation. The TPS grid is a mathematically formalized equivalent to the Cartesian transformation grid (CTG) introduced in 1917 by D'Arcy Thompson, a technique which requires construction by eye, brain, and hand.

TPS is used here to "replicate" Edwin Colbert's 1935 CTG analysis of shape change from Subhyracodon occidentalis, a primitive fossil rhino, to Rhinoceros unicornis, a derived living form. Twenty-two landmarks were defined on Colbert's original line drawings and coordinate data was retrieved by digitization. TPS analysis of this data was nearly instantaneous using an $8038620 \mathrm{MHz}$ PC.

TPS mapping of $S$. occidentalis onto $R$. unicornis produces a transformation similar to Colbert's CTG (differing most at the mandibular angle) and reveals both global and local aspects of the shape change. The global changes are dorsoventral heightening and rostrocaudal shear. Local changes include rostrocaudal shortening in the occipital and preorbital regions and rotations of the occiput and rostrum toward each other (producing the saddle-shaped skull of R. unicornis).

The functional consequences of these changes may be interrelated. For example, the upward and forward displacement of the occiput in $R$. unicornis suggests a change in head posture (nuchal attachment and range of motion) and a change in masticatory processing (increased vertical action of the temporalis muscle). Both functional changes may relate to dietary preference.

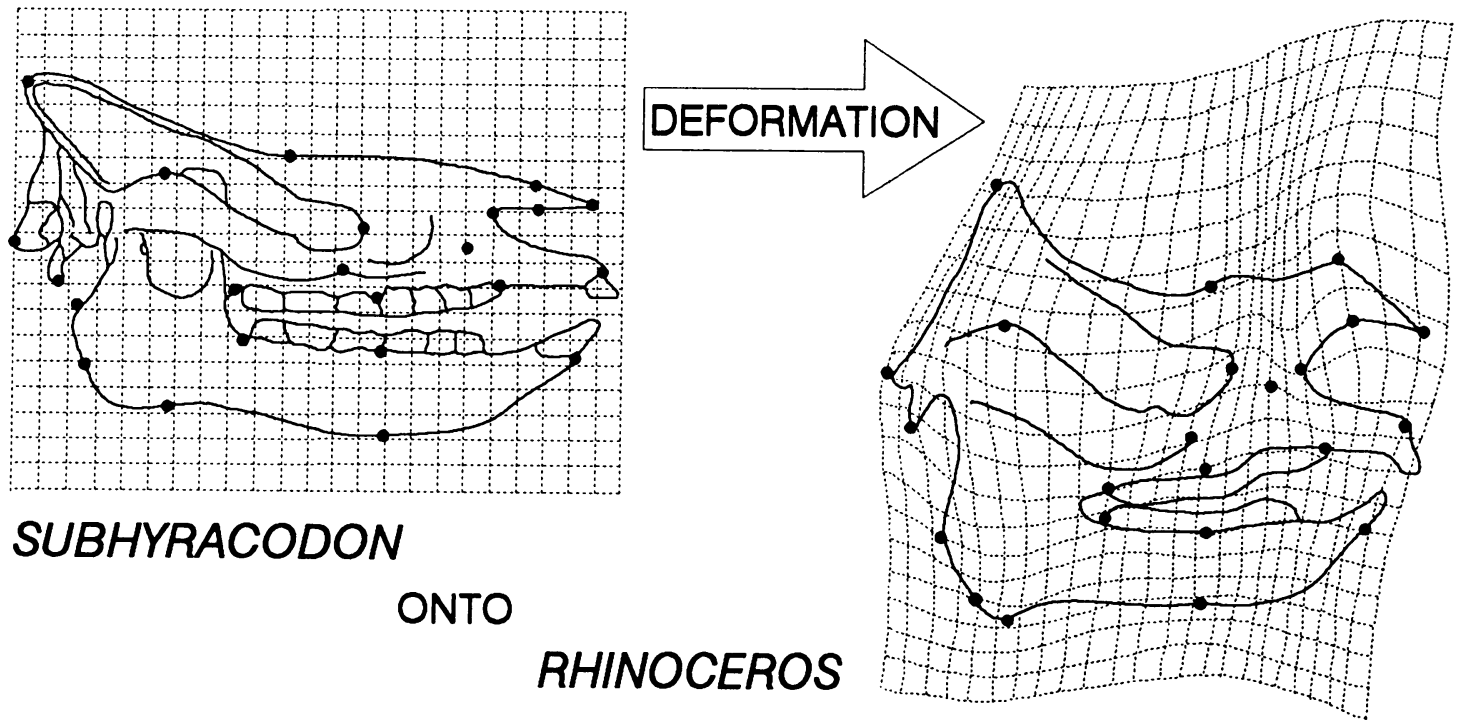

